\title{
Laryngeal mask cuff inflation at removal does not affect early postoperative laryngopharyngeal morbidity
}

\author{
[Un ballonnet encore gonflé lors du retrait du masque laryngé ne modifie pas la \\ morbidité laryngo-pharyngée postopératoire]
}

Brian O' Brien FCARCSI, Dominic Harmon FCARCSI, Michelle Duggan FFARCSI, Noel Flynn MD

Purpose: We assessed the effect of cuff inflation of the laryngeal mask airway at removal on sore throat, pharyngeal morbidity and airway complications.

Methods: In a prospective randomized trial, we used a standardized technique of anesthesia and of laryngeal mask insertion in 126 consecutive day-case patients. Postoperatively, on eye opening, the masks were removed either inflated (Group A) or deflated (Group B) and examined for blood by a blinded observer. Episodes of coughing, gagging, laryngospasm, hiccups and retching, and symptoms of sore throat and hoarseness were recorded by the same observer.

Results: Demographics were similar. Bloodstaining occurred in $21 \%$ of patients in Group A $(n=63)$ vs 13\% in Group B $(n=63$; $P=0.23)$; the incidence of sore throat was identical (19\%). Group A experienced more hoarseness (22\% vs $9 \% ; P=0.05)$. Overall airway complications did not differ between groups (19\% vs I I\%; $P=0.21)$.

Conclusion: We conclude that removal of the laryngeal mask airway inflated does not reduce the incidence of sore throat, pharyngeal morbidity or airway complications.

Objectif : Évaluer l'effet du gonflement du ballonnet du masque laryngé lors du retrait sur l'incidence de maux de gorge, sur la morbidité pharyngée et sur les complications touchant les voies respiratoires.

Méthode : Au cours d'un essai prospectif et randomisé, nous avons utilisé une technique normalisée d'anesthésie et d'insertion du masque laryngé chez 126 patients consécutifs en chirurgie d'un jour. Après l'opération, à l'ouverture des yeux, le masque était enlevé, soit en gardant le ballonnet gonflé (Groupe A), soit non gonflé (Groupe B) et examiné pour la présence possible de sang par un observateur impar- tial. Les épisodes de toux, de haut-le-cœur, laryngospasme et efforts pour vomir, et les symptômes de maux de gorge et de raucité de la voix ont été notés par le même observateur.

Résultats : Les données démographiques des patients étaient comparables. Des traces de sang ont été observées chez $21 \%$ des patients du Groupe A $(n=63)$ vs $13 \%$ du Groupe B $(n=63 ; P=$ $0,23)$; l'incidence de mal de gorge a été identique (19\%). On a noté plus d'enrouement chez les patients du Groupe A (22\% vs $9 \%$; $P=$ $0,05)$. L'ensemble des complications n'a pas présenté de différence intergroupe (19\% vs $11 \%$; $P=0,21$ ).

Conclusion : Le retrait du masque laryngé dont le ballonnet est encore gonflé ne réduit pas l'incidence de mal de gorge, de morbidité pharyngée ou de complications des voies respiratoires.

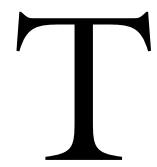

HE laryngeal mask airway (LMA) is widely used in anesthesia to maintain a patent airway in fasting patients undergoing day-case and routine surgery. During anesthesia the cuff is usually kept inflated; 1 at the conclusion of the procedure practices differ as some anesthesiologists deflate it prior to removal while others remove the device with the cuff inflated. Advocates of the former approach suggest that the smaller deflated cuff should cause less trauma to the pharynx, while the latter approach, it is argued, by bearing out more secretions should evoke less coughing, and other complications. Studies have mostly evaluated the effect of initial and intraoperative cuff volumes on laryngopharyngeal morbidity, ${ }^{2}$ though it has been established that the removal of the device in an inflated state removes a greater mass

From the Department of Anesthesia, University College Hospital, Galway, Ireland.

Address correspondence to: Dr. Brian O'Brien, Department of Intensive Care Medicine, The Alfred Hospital, Commercial Rd., Prahran

3181, Victoria, Australia. Phone: 613-92763036; Fax: 613-92763780; E-mail: drbobrien@hotmail.com

Note: No grants or financial support were received by the authors in the conduct of this study.

Accepted for publication April 11, 2002.

Revision accepted July 10, 2002. 
of secretions. ${ }^{3}$ Whether this is clinically significant remains unclear. Since sore throat following LMA usage is experienced by about one third of patients in the postanesthesia care unit, ${ }^{4}$ it seems appropriate to study the clinical impact cuff inflation during removal may have on this complication. The aim of this study then was to assess the influence of the inflationary state of the LMA at removal on clinical postoperative pharyngeal morbidity and airway complications.

\section{Methods}

Following local Ethics Committee approval, 126 consecutive adult patients undergoing general anesthesia for minor surgery using the LMA were included. A priori power analysis determined that a sample size of 54 patients in each group would be able to detect a $25 \%$ absolute difference (from 35\% to 10\%) between groups of sore throat following LMA use during general anesthesia $(\alpha=0.05)$, with a power of $80 \%$. Patients were excluded from the analysis if more than one attempt was required to insert the LMA, there was history of recent sore throat or of respiratory infection.

Premedication was not given, anticholinergic agents were avoided and anesthetic management was standardized. Standard monitors of electrocardiogram, pulse oximetery, capnography and non-invasive blood pressure were applied prior to induction of anesthesia. Patients were preoxygenated for three minutes. Anesthesia was induced with propofol 2.5 $\mathrm{mg} \cdot \mathrm{kg}^{-1}$ and fentanyl $1.5 \mu \mathrm{g} \cdot \mathrm{kg}^{-1}$ followed by $\mathrm{N}_{2} \mathrm{O}$ and sevoflurane in oxygen. All patients had an LMA inserted using a standardized approach (with the posterior aspect of the LMA against the hard palate and a single twisting movement) with the cuff partly inflated $(10 \mathrm{~mL}$ in size $3,20 \mathrm{~mL}$ in size 4$)$ and lubricant on the lateral and posterior aspects. Size 3 masks were used in women and size 4 masks in men. The masks were assessed clinically by easy ventilation of the lungs without a significant leak at $15 \mathrm{~cm} \mathrm{H}_{2} \mathrm{O}$ and by a nonobstructed capnography trace. Masks were placed by two experienced anesthesiologists unaware of patient group (this was only decided at the conclusion of the procedure by opening of sealed envelopes). No attempt was made to ventilate the patients' lungs by face mask or to instrument the airway with suction catheters at any time during the anesthetic. Diclofenac $100 \mathrm{mg} p r$ was administered with the patients' prior consent and the wound infiltrated with local anesthetic where appropriate, to provide postoperative analgesia. Intraoperative airway complications were recorded, the intraoperative period being considered to end when the sevoflurane and nitrous oxide were turned off at the conclusion of surgery.
Group A patients $(n=63)$ had their LMAs removed inflated, and Group B theirs removed deflated $(n=63)$. LMAs were removed by the attending anesthesiologist when the patient was able to open his/her mouth to command and were inspected by a blinded observer (the recovery room nurse) for the presence of blood. All masks were presented inflated for examination. The same nurse observer then asked the patient about the symptoms of sore throat and hoarseness and recorded episodes of hiccup, retching, laryngospasm and coughing/gagging while in the recovery room. Nurses were randomly allocated to patients, as is our usual hospital practice. Continuous data were analyzed using student's t test; frequency data were analyzed using the Chi square test with 95\% confidence intervals $(\mathrm{CI})$. A $P$ value $<0.05$ was considered statistically significant.

\section{Results}

One hundred and twenty-six patients participated in the study over a nine-month period, from March until December of 2000. The groups did not differ significantly in their demographics, duration of surgery or smoking behaviour (Table I). Intraoperative airway complications of hiccupping, coughing or gagging were seen in $13(10 \%)$ patients. Five $(8 \%)$ patients in Group A had intraoperative airway complications (i.e., three episodes of hiccupping and two of gagging and coughing) as did eight (12\%) patients in Group B (six episodes of coughing and gagging, and two of hiccups). These differences were not statistically significant $(P=0.38)$.

Postoperative airway complications did not differ significantly between groups (Group A having 12 cases $(19 \%)$ vs seven in Group B $(11 \% ; P=0.21,95 \%$ CI, 0.20-0.04). In this group, there was one postoperative laryngospasm - the other 11 events were combinations of coughing and gagging. In Group B, six of the seven postoperative complications were of coughing and gagging, while one was a case of hiccups (Table II). Thirteen (21\%) patients had blood stained masks in Group B $(n=63)$ vs eight $(13 \%)$ patients in Group A $(n=63 ; P=0.23,95 \%$ CI, 0.04-0.20). The incidence of sore throat was the same in both groups (19\%). There was an increased incidence of hoarseness in Group A i.e., $14(22 \%)$ vs six $(9 \%),(P=0.05,95 \%$ CI, 0.01-0.25).

\section{Discussion}

In the present study we found a low incidence of symptomatic pharyngeal morbidity and airway complications in a group of 126 patients as would be anticipated. The incidence of sore throat associated with LMA usage has 
TABLE I Patient characteristics and durations of surgical procedures

\begin{tabular}{llll}
\hline & Group A & Group B & P value \\
\hline Age $(\mathrm{yr})$ & $41.6(18-72)$ & $38.7(18-83)$ & 0.33 \\
Weight $(\mathrm{kg})$ & $72.9(15.3)$ & $71(15.2)$ & 0.49 \\
Duration $(\mathrm{min})$ & $33(26.0)$ & $38(30.3)$ & 0.49 \\
Gender $(\mathrm{m} / \mathrm{f})$ & $30 / 33$ & $26 / 37$ & 0.47 \\
Smoker $(\mathrm{y} / \mathrm{n})$ & $30 / 33$ & $28 / 35$ & 0.72 \\
\hline
\end{tabular}

Values are means (standard deviation or range). Age, weight and duration were compared using Student's unpaired t test, and gender and smoking using the Chi squared test.

TABLE II The incidence of intraoperative and postoperative airway complications in Group A (with cuff inflated at removal) and Group B (deflated at removal)

\begin{tabular}{lll}
\hline & Group $A(n=63)$ & Group $B(n=63)$ \\
\hline Intraoperative complications & 5 & 8 \\
Gagging/coughing & 2 & 6 \\
Hiccupping & 3 & 2 \\
Laryngospasm & 0 & 0 \\
Retching & 0 & 0 \\
Recovery room complications & 12 & 7 \\
Gagging/coughing & 11 & 6 \\
Hiccupping & 0 & 0 \\
Laryngospasm & 1 & 0 \\
Retching & 0 & 0 \\
Blood on LMA & 8 & 13 \\
Sore throat & 12 & 12 \\
Hoarseness & $14^{*}$ & 6 \\
\hline
\end{tabular}

The operative period is taken to end when the delivery of anesthetic vapour ceases. Values shown are patient numbers. Statistical significance (where $P=0.05$, in this case) is denoted as * ${ }^{*} \mathrm{LMA}=$ laryngeal mask airway.

been found to vary widely from 0 to $50 \%{ }^{5}$ That of early postoperative sore throat (i.e., in the recovery room) has been found to be in the region of $35 \% ;$; though our power analysis was based on this figure, our observed rate of sore throat was a little lower. Our results are also compatible with the low rate of clinical complications observed in the 100 million or more uses of the device worldwide since its introduction. ${ }^{2}$ We found no significant relationship between the presence of air in the cuff at the time of removal and patient outcome or bloodstaining of the device.

Our protocol involved the use of LMAs one size smaller than recommended by a variety of investigators, as discussed in a recent review. ${ }^{2}$ However, as those authors observed, "the size 3 mask seems to be used frequently in females and the size 4 in males. The reasons for this practice are not clear". This remains more common practice in our institution, although increasingly the larger sized LMAs are now being used. For the purposes of the study we chose to use the more familiar, smaller sized masks, however.

Whilst initially it was recommended that the LMA be deflated prior to removal, subsequent guidelines suggested that the device be removed with the cuff moderately inflated "to aid more complete removal of salivary secretions". ${ }^{6}$ It is indeed established that a greater quantity of secretions is thus removed; ${ }^{3}$ however it appears from our findings that no clinical benefit accrues form this approach. This is in keeping with the suggestion of Deakin and colleagues ${ }^{3}$ that while "the difference in the mass of secretions removed is statistically significant, we do not believe (it) is likely to be clinically significant". We have found the patient outcome to be similar and suggest that the decision whether to remove the LMA inflated or deflated is not clinically important, as airway and laryngopharyngeal morbidity are uncommon, and appear to be unaffected by the decision.

\section{References}

1 Morris GN, Marjot R. Laryngeal mask airway performance: effect of cuff deflation during anaesthesia. Br J Anaesth 1996; 76: 456-8.

2 Asai T, Brimacombe J. Cuff volume and size selection with the laryngeal mask. Anaesthesia 2000; 55: 1179-84.

3 Deakin CD, Diprose P, Majumdar R, Pulletz M. An investigation into the quantity of secretions removed by inflated and deflated laryngeal mask airways. Anaesthesia 2000; 55: 478-80.

4 Brimacombe J, Holyoake L, Keller C, et al. Emergence characteristics and postoperative laryngopharyngeal morbidity with the laryngeal mask airway: a comparison of high versus low initial cuff volume. Anaesthesia 2000; 55: 338-43.

5 Keller C, Sparr HJ, Brimacombe JR. Laryngeal mask lubrication. A comparative study of saline versus $2 \%$ lignocaine gel with cuff pressure control. Anaesthesia 1997; 52: 592-602.

6 Brimacombe JR, Brain AIJ, Berry AM. The Laryngeal Mask Airway Instruction Manual. $4^{\text {th }}$ ed. London: Intavent Research Ltd., 1999. 